\title{
石墨烯衍生物作为无金属碳基催化剂在有机催化中的应用
}

\author{
黄杰奚江波* 陈伟* 柏正武 \\ (武汉工程大学 化学与环境工程学院 绿色化工过程教育部重点实验室 武汉 430205)
}

\begin{abstract}
摘要 近年来, 随着绿色化学和可持续发展等创新理念的提出, 无金属催化剂逐渐成为催化领域的研究热点和前沿. 石墨烯作为一种新型纳米碳材料, 具有机械强度大、比表面积高、稳定性好、电学性质优异等特点, 经过改性或功能 化后的衍生物可以作为无金属碳基催化剂，在有机反应中展现了良好的应用前景. 本文综述了石墨烯衍生物的结构和 性质, 探究了石墨烯基材料的结构与催化活性之间的关系, 总结了此类材料作为无金属催化剂在氧化、还原/氢化、偶 联、取代反应以及其他有机反应中的应用和反应机理.
\end{abstract}

关键词 石墨烯; 石墨烯衍生材料; 碳基催化剂; 有机反应; 无金属催化; 催化机理

\section{Graphene-derived Materials for Metal-free Carbocatalysis of Organic Reactions}

\author{
Jie Huang Jiangbo Xi* Wei Chen* Zhengwu Bai \\ (School of Chemistry and Environmental Engineering, Key Laboratory of Green Chemical Engineering Process of \\ Ministry of Education, Wuhan Institute of Technology, Wuhan 430205, China)
}

\begin{abstract}
The rise in global demand for green chemistry and sustainable development have driven immense research in the fundamental science of catalysis. Metal-free catalysis, serves as an ideal industrial technology, has emerged as a new frontier and hot point in modern catalysis science due to its green and sustainable properties. As a new carbonaceous material, graphene exhibits high surface area, excellent stability, unique mechanical and electronic properties. Its physical and chemical properties can be further modulated by modification and functionalization. Graphene derivatives have been successfully employed in a variety of chemical transformations recently, showing them to be promising metal-free organocatalysts. This review highlights the recent advancements of graphene-derived materials as metal-free carbocatalysts and their application in organic reactions, such as oxidation, reduction or hydrogenation, coupling, substitution, etc. The possible structure-performance relationships of graphene-based carbocatalysts and metal-free organocatalytic mechanisms are also emphasized.
\end{abstract}

Keywords graphene; graphene-derived material; carbocatalyst; organic reaction; metal-free catalysis; catalytic mechanism

\section{1 研究背景}

催化是自然界中普遍存在的现象, 在现代化学工业 中，据统计约有 $90 \%$ 的化学产品在生产过程中都使用了 催化剂 ${ }^{[1]}$. 活性高、选择性强、稳定性好、回收简便的 催化剂，在降低原材料和能源的消耗、提高生产效率、 改进产品质量、防止环境污染等方面都起着重要的作用. 目前, 大部分均相和非均相催化反应都使用了基于金属 的催化剂 ${ }^{[2]}$, 如金属盐 ${ }^{[3]}$ 、金属配合物 ${ }^{[4]}$ 、分子篮 ${ }^{[5]}$ 、金 属氧化物 ${ }^{[6]}$ 以及商业金属/碳类催化剂[7]等. 然而, 金属 尤其是贵金属存在资源有限、价格高、易失活和后处理 困难(后处理经济成本高和容易导致二次污染)等弊端, 这些都成为制约金属基催化剂工业应用和发展的瓶颈 问题 ${ }^{[8-10]}$.
近年来，随着绿色化学和可持续发展等概念的提出 和发展, 人们对催化剂的催化性能、使用成本、环境友 好性以及循环使用寿命等方面提出了更高的要求 ${ }^{[11]}$. 无 金属碳基催化剂(carbocatalyst)具有资源可再生、环境友 好、价格低廉、稳定性高和循环使用性好等优点，已经 受到催化领域科研工作者的高度关注. 从可持续发展和 经济成本的角度考虑，研发新型碳基无金属催化剂来替 代金属基催化剂将有助于缓解上述问题，并有望降低催 化行业对于金属的依赖. 因此, 研制具有高活性、高选 择性和高稳定性的碳基无金属催化剂将是未来催化工 业领域的重要发展方向, 不仅具有重大的科研价值, 也 具有良好的社会效益和广阔的应用前景.

碳材料在化学催化中的应用可以追溯到 20 世纪二 三十年代, Kutzelnigg 和 Kolthoff 在 1930 年报道了活性

*E-mail: jbxi@wit.edu.cn; wchen@wit.edu.cn; Tel.: 027-87195680.

Received July 23, 2021; published August 23, 2021.

Project supported by the National Natural Science Foundation of China (No. 51772110) and the Open Project of Key Laboratory of Green Chemical Engineering Process of Ministry of Education (No. GCP20200205).

国家自然科学基金(No. 51772110)和绿色化工过程教育部重点实验室开放基金(No. GCP20200205). 
炭可以将亚铁氰化物催化氧化为铁氰化物 ${ }^{[12]}$, 在 20 世 纪七八十年代, 碳材料被发现可以作为脱氢反应的催化 剂 [13]. 早期的碳催化材料主要是炭黑、活性炭或石墨, 与传统的金属或者金属氧化物催化剂相比, 具有很强的 抗酸碱能力, 但是它们的催化活性不够高 ${ }^{[14-15]}$. 石墨烯 是碳材料中的新星, 主要是由于它具有优异的电学、光 学、热学和力学性能, 以及高比表面积(理论值为 2630 $\mathrm{m}^{2} / \mathrm{g}$ ), 是一种非常有潜力的新型碳材料 [16-18]. 石墨烯中 的碳原子皆为 $\mathrm{sp}^{2}$ 杂化, 具有完美的六角形蜂巢二维晶 体结构 [19-20]. 然而, 也正是由于它规则和均匀的电子结 构弱化了其催化活性, 限制了其应用范围 [21]. 石墨炔是 继石墨烯之后发展的一种新型全碳纳米结构二维材料, 其独特的物化性质使得其在电催化领域展现了良好的 应用前景 ${ }^{[22-27]}$, 在有机催化性能方面的应用潜力还有待 进一步探索. 近年来, 随着石墨烯改性和功能化技术的 发展, 石墨烯衍生物也成为了催化研究领域的理想材料 之一[28-31]. 研究发现, 向石墨烯结构中引入杂原子可以 改变与之相邻碳原子的电荷分布, 调控其物理和化学性 质, 创造新的催化活性位点 ${ }^{[32]}$. 向石墨烯中掺杂氮原子 可以在石墨烯表面诱导形成高的局域电荷/自旋密度, 提高其化学活性, 掺杂磷原子可以增强碳原子的电荷离 域, 并造成更多边缘活性位点 ${ }^{[33]}$. 已有大量研究探究了 掺杂对石墨烯材料催化性能的影响，选择合理原子尺寸 和电负性的原子并有效地将其掺杂入石墨烯结构中, 从 而制备高性能掺杂石墨烯材料(图 1).

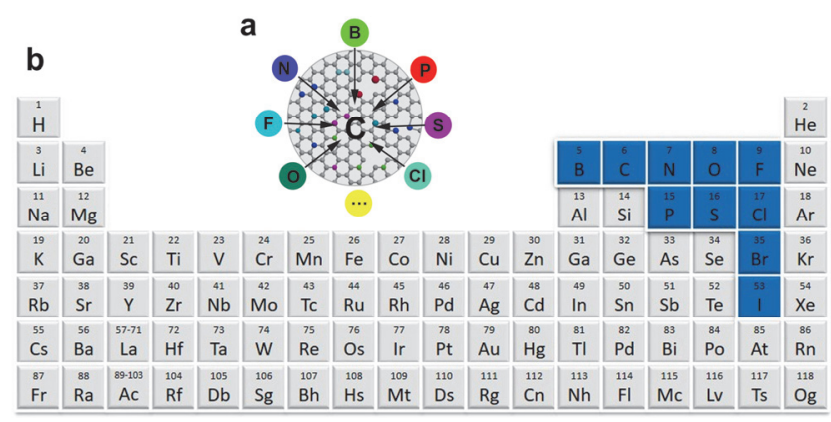

图 1 (a)在石墨烯中引入杂原子; (b)元素周期表及常见掺杂元素 Figure 1 (a) Doping of graphene with heteroatoms; (b) periodic table and the corresponding elements which can be doped into graphene

石墨烯基碳材料作为催化剂, 已经在氧化反应、还 原反应、偶联反应和取代反应等诸多有机反应中得以应 用, 弥补了传统碳材料催化性能弱和稳定性差的缺点, 而且对环境友好、易回收, 被认为是一类优质的 “绿色” 催化剂. 虽然近年来已有多篇文章报道了碳基材料在有 机催化领域的研究进展, 然而这些文献分别着重于讨论 一类反应或功能化石墨烯基材料的制备及其无金属催 化应用 ${ }^{[28,31,34]}$. 因此, 对于石墨烯基催化剂的制备方法、 有机化学反应类型、无金属催化机理等方面的最新进展, 仍有待更新并进行系统性总结和评述. 鉴于此, 本综述 对石墨烯衍生物的制备方法、活性位点、构效关系和无
金属催化机理等方面的最新进展进行了深入探讨和更 新补充. 首先综述了石墨烯及其衍生物的特性和制备方 法; 随后系统介绍了石墨烯基碳材料在常见的几类有机 反应中的催化性能和无金属催化机理, 并总结了其活性 位点; 同时，梳理了石墨烯基碳材料的结构与催化性能 的关系; 最后, 对石墨烯基碳材料在催化领域的应用前 景和挑战进行了展望.

\section{2 常见的石墨烯基碳材料及其特性}

\section{1 氧化石墨和氧化石墨烯(GO)}

氧化石墨是一种常用于制备石墨烯的前体材料，是 石墨经过氧化的产物. 氧化石墨首次由牛津大学的 Brodie 教授在 1859 年使用氯酸钾和发烟硝酸氧化石墨 制备，随后 Staudenmaier、Hofmann 和 Hummers 对该方 法进行了改进，使其更加简便和安全 ${ }^{[35]}$. 对氧化石墨进 行进一步剥离可以得到氧化石墨烯, 常用的剥离方法有 超声法、溶剂剥离法、热剥离法等. GO 是石墨烯的重要 衍生物, 不仅是氧化还原法制备石墨烯的前驱体，也是 制备其他石墨烯衍生物 (如杂原子掺杂石墨烯)的原 料 [36-37]. 目前, 制备氧化石墨和 GO 最常用的方法是 Hummers 法, 该方法以浓 $\mathrm{H}_{2} \mathrm{SO}_{4} 、 \mathrm{KMnO}_{4}$ 和 $\mathrm{NaNO}_{3}$ 作 为氧化剂[38-39]. 在氧化的过程中石墨的层状结构保持不 变, 石墨片被氧化表面形成大量含氧基团，从而导致层 与层距离扩大 ${ }^{[40]}$. 制备的 GO 表面和边缘含有丰富的羟 基、环氧基、羧基等含氧官能团, 使其更加易于进一步 加工和复合 ${ }^{[41]}$. 目前 GO 的精确结构在研究领域并没有 一致的结论, 大家普遍接受的 GO 模型结构是由 Lerf 和 Klinowski 提出的 ${ }^{[42-43]}$, 着基和环氧基随机分布在 GO 片 表面上, 羰基和羧基主要分布在 $\mathrm{GO}$ 片的边缘和褶皱处 (图 2a). 这些含氧基团的存在使得石墨烯骨架不再保持 电中性, 而是带有部分电荷 ${ }^{[12]}$. 此外, 含氧基团不仅使 GO 在水等极性溶剂中具有良好的分散性, 而且可以作 为催化反应的活性位点. 这使得 GO 可以作为固体酸参 与一些原本由常规酸催化的有机反应 ${ }^{[44]}$, 在氧化反应 中可作为氧气转化为氧自由基的位点 ${ }^{[32]}$, 在偶联反应 中则可作为亲核试剂. 此外, GO 上丰富的含氧官能团 可以作为反应的位点引入新的基团, 实现对 GO 的结构 修饰或改性. 如 GO 中的差基可以和异氭酸酯发生反应 得到氨基甲酸酯修饰的 $\mathrm{GO}, \mathrm{GO}$ 边缘的羒基可以与氯化 亚砜和二环已基碳二亚胺等试剂反应，进行结构修饰. 这些通过化学键构筑的修饰结构可调控 GO 的性质, 扩 展其应用范围 ${ }^{[45-48]}$.

\section{2 还原氧化石墨烯( $(\mathrm{GO})$}

对 GO 进行还原, 可以脱除 $\mathrm{GO}$ 表面的大部分含氧 基团, 得到含氧量较低的石墨烯, 如采用化学还原法制 备石墨烯，也被称为还原氧化石墨烯( $\mathrm{rGO})$. 氧化还原 法制备石墨烯的方法简单、可宏量制备、易于后续功能 

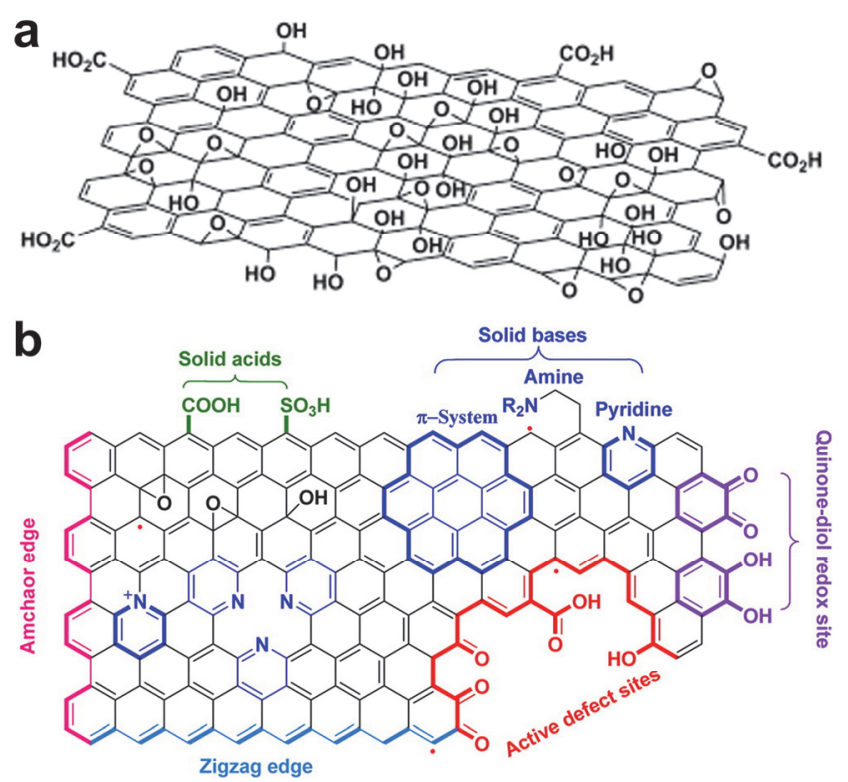

图 2 (a) GO 的结构模型 ${ }^{[3]}$; (b) 石墨烯基碳材料催化活性中心示意 图[49]

Figure 2 (a) Structure model of GO proposed by Lerf-Klinowski ${ }^{[43]}$; (b) schematic showing active catalytic sites in the graphene based materials $^{[49]}$

化或修饰. GO 常见的还原方法包括化学还原法、热还原 法或电化学法等 ${ }^{[50-51]}$. 由于含氧基团的还原和脱除, GO 的含氧量可由原子百分比 $20 \%$ 降低到 $\mathrm{rGO}$ 的 $5 \%{ }^{[44]}$, rGO 中的 $\pi$ 共轭程度也相应增加. $\mathrm{rGO}$ 的表面随之产生了大 量缺陷 ${ }^{[52]}$, 这可能是表面的含氧基团脱除时导致碳原 子缺失, 从而造成空洞 ${ }^{[53]}$. rGO 中的缺陷位点和含氧官 能团被认为是无金属催化反应的活性中心, 使 $\mathrm{rGO}$ 具备 催化活性 ${ }^{[54]}$. 此外, 石墨烯表面 $\pi$ 共轭结构有利于芳香 化合物的吸附与活化 ${ }^{[55]}$, 石墨烯的 zigzag 式或 armchair 边缘的不饱和碳原子呈现出特殊的电子态, 也可以作为 催化活性位点, 如图 2(b)所示 ${ }^{[56]}$.

\section{3 化学修饰石墨烯}

对石墨烯进行化学修饰是石墨烯基碳材料改性的 一种常用方法, 石墨烯基碳材料的适当修饰不仅可以避 免石墨烯片之间的堆叠, 而且可以增强其催化性能, 扩 大应用范围 ${ }^{[34]}$. 化学修饰的方式可以分为共价键修饰 和非共价作用修饰 ${ }^{[33]}$. 共价键修饰通过与石墨烯表面 的官能团形成共价键来实现, 这种方法改性的石墨烯具 有良好的稳定性，但是会破坏石墨烯的部分共轭体系， 降低石墨烯材料的导电性. 利用 GO 表面的含氧基团, 连接一些官能团就是一种常见的共价键修饰方式, Azlouk 等 ${ }^{[57]}$ 报道了首先在 $\mathrm{GO}$ 表面的羟基上连接 3-氨丙 基三乙氧基硅烷制备了胺功能化 $\mathrm{GO}\left(\mathrm{GO}-\mathrm{NH}_{2}\right)$, 然后通 过交联反应将 $L$-脯氨酸接枝在 $\mathrm{GO}-\mathrm{NH}_{2}$ 上得到了相应的 衍生物. 将制备的石墨烯衍生物作为 aldol 反应的非均 相手性催化剂, 产物的对映体过量可以达到 $85 \% e e$, 展 现了良好的立体选择性. 非共价键作用修饰则是指利用
修饰基团与石墨烯基面的相互作用力(如 $\pi-\pi$ 相互作用 和范德华力)来实现材料改性, 虽不破坏碳材料的结构, 但是其稳定性较差 ${ }^{[40]}$.

\section{4 掺杂石墨烯}

对石墨烯基碳材料进行杂原子(硼、氮、氧、磷、 硫或卤素等)掺杂是其改性的另一种常用方法，这种方 法可以突破材料本身的结构局限, 改变碳材料的表面性 质，减少石墨烯片层之间的堆叠，增强碳材料导电性. 而且, 由于杂原子与碳的电负性存在差异, 也导致碳材 料的电子结构发生变化 ${ }^{[58]}$. 相较于其它掺杂原子, 氮在 元素周期表中与碳相邻，二者原子半径近似，使氮原子 较容易掺杂入石墨烯骨架中, 因而对于石墨烯的氮掺杂 和应用研究也最为广泛 ${ }^{[59]}$. 此外, 由于氮的电负性大于 周围的碳原子, 可以使周围的碳原子显示正电性, 有利 反应中物质的吸附促进反应的进行 ${ }^{[60]}$. 随着对掺杂碳 材料研究的不断深入, 多种杂原子共同掺杂的石墨烯基 材料也被制备出来，并实现无金属催化性能的提 升 ${ }^{[61-63]}$. 由于不同的杂原子的原子半径和电负性都存在 差别, 在石墨烯中同时掺杂多种元素可以导致碳骨架形 成独特的电子结构, 产生特殊的协同效应, 进而实现石 墨烯基材料催化性能的调控 ${ }^{[64]}$. 由于石墨烯衍生物的 制备方法多种多样, 所制备的石墨烯基催化剂的元素组 成和官能团也不尽相同，进而可以应用于多种类型的有 机反应体系中. 为了进行简明扼要的概括, 我们将这些 内容总结列于表 1 中.

\section{3 石墨烯基碳材料在有机反应中的催化应用}

在这一部分我们主要综述石墨烯基无金属催化剂 在氧化反应、还原反应、偶联反应和取代反应等经典反 应中的应用, 并且介绍参与催化反应的可能活性位点 (如表 2). 石墨烯基碳材料表面的 $\pi$ 共轭结构、含氧基团、 缺陷、未成对电子对以及掺杂原子都可以作为催化反应 的活性位点, 在反应中它们可以吸附激活底物或者有利 于活性中间态的稳定来促进反应的进行.

\section{1 氧化反应}

氧化反应是有机合成中重要的反应之一, 化工生产 常用到的醛、酮、酸等化合物都可以通过氧化反应制 备 ${ }^{[65]}$. 石墨烯材料在此类反应中展现出了良好催化性 能, 可以作为制备醛酮、环氧化物、苯酚等化合物的催 化剂. 2010 年, Bielawski 及其同事[66]发现 GO 可以催化 苯甲醇和环己醇等醇类化合物的空气氧化, 制备相应的 羰基化合物，苯甲醛的产率可以达到 $92 \%$. 这是石墨烯 基材料作为碳基无金属催化剂参与有机合成反应的首 次报道，为其他石墨烯基碳材料在有机合成中的应用打 开了大门. 为了阐明其无金属催化机理, Bielawski 及其 合作者 ${ }^{[67]}$ 采用密度泛函理论(DFT)进行了深入研究, 理 论计算结果表明 GO 表面的环氧官能团可能是反应的活 
表 1 石墨烯基催化剂的制备、组成和适用的有机反应

Table 1 Preparation method, composition of graphene-based carbocatalysts and their applications in organic reactions

\begin{tabular}{|c|c|c|c|}
\hline Catalyst & Preparation method & Functional groups or dopants & Reaction \\
\hline $\mathrm{GO}^{[66]}$ & Hummers method & O-containing groups & \multirow{5}{*}{ Oxidation of alcohol } \\
\hline $\mathrm{abGO}^{[68]}$ & GO was treated with sequential base and acid & O-containing groups & \\
\hline $\mathrm{NG}-\mathrm{T}^{[69]}$ & GO annealed under $\mathrm{NH}_{3}$ flow & Doped $\mathrm{N}$ atoms & \\
\hline $\mathrm{BG}^{[70]}$ & GO annealed with boric acid & Doped B atoms $(2.9 \%)$ & \\
\hline $\mathrm{G} 1000^{[71]}$ & Pyrolysis of the sulfate lignin & Doped $\mathrm{S}$ and $\mathrm{O}$ atoms & \\
\hline $\mathrm{CCG}^{[55]}$ & Chemical reduction of GO & $\pi$-system of graphene & Oxidation of benzene \\
\hline $\mathrm{GO}^{[72]}$ & Modified Hummers method & O-containing groups & Oxidation of cyclohexane \\
\hline $\mathrm{NGO}^{[73]}$ & Modified Hummers method & O-containing groups & \multirow{2}{*}{$\begin{array}{l}\text { Oxidation of alkyl-substituted } \\
\text { arenes }\end{array}$} \\
\hline $\mathrm{LC}-\mathrm{N}^{[74]}$ & CVD method with acetonitrile as $\mathrm{N}$ source & Doped $\mathrm{N}$ atoms $(8.9 \%)$ & \\
\hline $\mathrm{NG}^{[75]}$ & $\begin{array}{l}\text { Arc-discharge exfoliation and subsequently annealed } \\
\text { with } \mathrm{NH}_{3}\end{array}$ & Doped $\mathrm{N}$ atoms $(0.42 \%)$ & Oxidation of olefin \\
\hline $\mathrm{rGO}^{[76]}$ & GO reduction (modified Wallace's method) & The zigzag edges of rGO & \multirow{4}{*}{ Reduction of nitroaromatics } \\
\hline $\mathrm{NG}^{[77]}$ & $\begin{array}{l}\text { Hydrothermal treatment of GO-urea mixture and subse- } \\
\text { quent annealing }\end{array}$ & Doped N atoms $(2.03 \%)$ & \\
\hline $\mathrm{NHG}^{[78]}$ & Hydrothermal treatment of GO- $\mathrm{NH}_{3}-\mathrm{H}_{2} \mathrm{O}_{2}$ mixture & Doped $\mathrm{N}$ atoms $(9.77 \%)$ & \\
\hline $\mathrm{NPG}^{[79]}$ & $\begin{array}{l}\text { Pyrolysis of a microwave-exfoliated graphite and hexa- } \\
\text { chlorocyclotriphosphazene }\end{array}$ & Doped $\mathrm{N}$ and $\mathrm{P}$ atoms & \\
\hline Graphene ${ }^{[80]}$ & Pyrolysis of alginate & Lewis acid-base pairs & Selective acetylene hydrogenation \\
\hline $\mathrm{GO}^{[81]}$ & Modified Hummers method & O-containing groups & \multirow{2}{*}{ Cross-coupling reaction } \\
\hline $\mathrm{GO}^{[82]}$ & - & O-containing groups & \\
\hline $\begin{array}{l}\text { Graphite } \\
\text { oxide }^{[83]}\end{array}$ & Modified Hummers method & O-containing groups & \multirow{5}{*}{ Oxidative coupling of amines } \\
\hline $\mathrm{ba}^{-\mathrm{GO}^{[84]}}$ & GO was treated with base and acid & O-containing groups & \\
\hline $\mathrm{PG}^{[85]}$ & Annealing of triphenylphosphine & Doped $\mathrm{P}$ atoms & \\
\hline $\mathrm{BNHG}^{[62]}$ & Annealing of of dicyandiamide, glucose and boric acid & Doped $\mathrm{B}$ and $\mathrm{N}$ atoms & \\
\hline $\mathrm{GO}^{[86]}$ & Modified Hummers method & O-containing groups & \\
\hline $\mathrm{GO}^{[87]}$ & Modified Hummers method & O-containing groups & \multirow[t]{2}{*}{ Friedel-Crafts alkylation } \\
\hline $\mathrm{GO}^{[88]}$ & Hummers method & O-containing groups & \\
\hline Graphite $^{[89]}$ & - & - & Friedel-Crafts acylation \\
\hline $\mathrm{GR}-\mathrm{SO}_{3} \mathrm{H}^{[90]}$ & $\begin{array}{l}\text { rGO reacts with sodium nitrite and sulfanilic acid at } \\
\text { room temperature }\end{array}$ & Denzenesulfonic acid groups & Transesterification \\
\hline GO-S $\mathrm{S}^{[91]}$ & Modified Hummers method & $\begin{array}{l}\text { Sulfonic acid groups and } \\
\text { O-containing groups }\end{array}$ & Esterification \\
\hline $\begin{array}{l}\text { Graphite } \\
\text { oxide }^{[92]}\end{array}$ & Hummers method & - & Claisen-Schmidt condensation \\
\hline GO-DETA $^{[93]}$ & GO modification with triethylamine & $\begin{array}{l}\text { Primary, secondary amino and } \\
\text { Oxygen-containing groups }\end{array}$ & $\begin{array}{l}\text { Michael reaction and Knoevenagel } \\
\text { reaction }\end{array}$ \\
\hline $\mathrm{GO}^{[94]}$ & Modified Hummers method & Oxygen-containing groups & Hydrolysis reaction \\
\hline $\mathrm{GO}^{[95]}$ & - & O-containing groups & $N$-formylation reaction \\
\hline $\mathrm{GO}^{[96]}$ & - & O-containing groups & Dehydrogenation reaction \\
\hline $\mathrm{GO}^{[97]}$ & - & O-containing groups & Iodization reaction \\
\hline $\begin{array}{l}\text { Graphite } \\
\text { oxide }^{[98]}\end{array}$ & Hummers method & O-containing groups & Dehydration reaction \\
\hline Graphite $^{[99]}$ & - & - & \\
\hline
\end{tabular}

表 2 基于石墨烯的碳基催化剂在有机反应中催化应用

Table 2 Graphene-based carbocatalysts for organic reactions and their probable active sites

\begin{tabular}{|c|c|c|c|c|}
\hline Reaction & Catalyst & Substrate & Product & Probable active site \\
\hline \multirow{5}{*}{ Oxidation of alcohol } & $\mathrm{GO}^{[66]}$ & Alcohol & Ketone/aldehyde & Epoxide groups \\
\hline & $\mathrm{abGO}^{[68]}$ & Benzylic alcohol & Benzaldehyde & Phenol hydroxyl groups \\
\hline & NG-T ${ }^{[69]}$ & Benzylic alcohol & Benzaldehyde & Graphitic $\mathrm{sp}^{2}-\mathrm{N}$ sites \\
\hline & $\mathrm{BG}^{[70]}$ & Benzylic alcohol & Benzaldehyde & $\mathrm{B}$ doping species $\left(\mathrm{BC}_{3}\right)$ \\
\hline & $\mathrm{G} 1000^{[71]}$ & Benzylic alcohol & Benzaldehyde & Doped $\mathrm{S}$ and $\mathrm{O}$ atoms \\
\hline Oxidation of benzene & $\mathrm{CCG}^{[55]}$ & Benzene & Phenol & $\begin{array}{l}\pi \text {-system of graphene and } \\
\text { O-containing groups }\end{array}$ \\
\hline Oxidation of cyclohexane & $\mathrm{GO}^{[72]}$ & Cyclohexane & $\begin{array}{l}\text { Cyclohexanone } \\
\text { Adipic acid }\end{array}$ & Carboxylic acid groups \\
\hline Oxidation of alkyl- & $\mathrm{NGO}^{[73]}$ & Toluene & Benzoic acid & - \\
\hline substituted arenes & $\mathrm{LC}-\mathrm{N}^{[74]}$ & Ethylbenzene & Acetophenone & $\mathrm{C}$ atoms adjacent to $\mathrm{N}$ atoms \\
\hline Oxidation of olefin & $\mathrm{NG}^{[75]}$ & Olefin & Alkylene oxide & Graphite N \\
\hline \multirow{2}{*}{ Reduction of nitroaromatics } & $\mathrm{rGO}^{[76]}$ & Nitrobenzene & Aniline & Edge sites \\
\hline & $\mathrm{NG}^{[77]}$ & Nitroaromatics & Aromatic amine & Graphitic N \\
\hline
\end{tabular}




\begin{tabular}{|c|c|c|c|c|}
\hline Reaction & Catalyst & Substrate & Product & Probable active site \\
\hline & $\mathrm{NHG}^{[78]}$ & Nitroaromatics & Aromatic amine & Doped $\mathrm{N}$ atoms \\
\hline & $\mathrm{NPG}^{[79]}$ & Nitroaromatics & Aromatic amine & Doped $\mathrm{N}$ and $\mathrm{P}$ atoms \\
\hline Selective hydrogenation & Graphene $^{[80]}$ & $\begin{array}{l}\text { Acetylene } \\
\text { Ethylene }\end{array}$ & $\begin{array}{l}\text { Ethylene } \\
\text { Ethane }\end{array}$ & Lewis acid-base pairs \\
\hline Cross-coupling reaction & $\mathrm{GO}^{[81]}$ & $\begin{array}{l}\text { Iodobenzene } \\
\text { Benzene }\end{array}$ & Biaryl compounds & O-containing groups \\
\hline \multirow{4}{*}{$\begin{array}{l}\text { Oxidative coupling of } \\
\text { amines }\end{array}$} & $\begin{array}{l}\text { Graphite } \\
\text { oxide }^{[83]}\end{array}$ & Benzylamine & $N$-Benzylidene aniline & - \\
\hline & ba-GO ${ }^{[84]}$ & Benzylamine & $N$-Benzylidene aniline & The edge sites carboxyl \\
\hline & $\mathrm{PG}^{[85]}$ & Benzylamine & $N$-Benzylidene aniline & Doped of $\mathrm{P}$ atoms \\
\hline & $\mathrm{BNHG}^{[62]}$ & $\begin{array}{l}\text { Benzylamine } \\
\text { Aromatics }\end{array}$ & $N$-Benzylidene aniline & Doped B and $\mathrm{N}$ atoms \\
\hline \multirow{3}{*}{ Friedel-Crafts alkylation } & $\mathrm{GO}^{[86]}$ & $\begin{array}{l}\text { Styrene } \\
\text { Alcohol }\end{array}$ & Diarylalkane & O-containing groups \\
\hline & $\mathrm{GO}^{[87]}$ & $\begin{array}{l}\text { Aldehydes } \\
\text { Indoles }\end{array}$ & Bis(indolyl)methanes & O-containing groups \\
\hline & $\mathrm{GO}^{[88]}$ & $\begin{array}{l}\text { Indole } \\
\text { Ethers }\end{array}$ & 3,3'-Bisindolylmethane & - \\
\hline Friedel-Crafts acylation & Graphite $^{[89]}$ & Aromatic compounds & Acylated products & - \\
\hline Transesterification & GR- $\mathrm{SO}_{3} \mathrm{H}^{[90]}$ & Palm oil & Biodiesel & Benzenesulfonic acid groups \\
\hline Esterification & $\mathrm{GO}-\mathrm{S}^{[91]}$ & Oleic acid & Biodiesel & $\begin{array}{l}\text { Sulfonic acid groups and carboxylic } \\
\text { acid groups }\end{array}$ \\
\hline $\begin{array}{l}\text { Claisen-Schmidt } \\
\text { Condensation }\end{array}$ & $\begin{array}{l}\text { Graphite } \\
\text { oxide }^{[92]}\end{array}$ & $\begin{array}{l}\text { Phenylacetylene } \\
\text { Benzyl alcohol }\end{array}$ & Chalcone & - \\
\hline $\begin{array}{l}\text { Michael reaction } \\
\text { Knoevenagel reaction }\end{array}$ & GO-DETA $^{[93]}$ & $\begin{array}{l}\text { Benzaldehyde } \\
\text { Malononitrile } \\
(E) \text {-Chalcone }\end{array}$ & $\begin{array}{l}\text { Benzylidenemalononitrile } \\
\text { 2-(3-oxo-1,3-diphenylpropyl) } \\
\text { malononitrile }\end{array}$ & $\begin{array}{l}\text { Primary, secondary amino and } \\
\text { carboxyl groups }\end{array}$ \\
\hline Hydrolysis reaction & $\mathrm{GO}^{[94]}$ & Cellulose & Glucose & Hydroxyl and carboxyl groups \\
\hline $\mathrm{N}$-formylation reaction & $\mathrm{GO}^{[95]}$ & Tetrahydroisoquinoline & Formamide & Carboxylic acid group \\
\hline \multirow{2}{*}{ Dehydration reaction } & $\begin{array}{l}\text { Graphite } \\
\text { oxide }^{[98]}\end{array}$ & Benzyl alcohol & Poly(phenylene methylene) & O-containing groups \\
\hline & Graphite $^{[99]}$ & $\begin{array}{l}\text { Ethanol } \\
\text { Silver nitrate }\end{array}$ & Silver cyanide & - \\
\hline
\end{tabular}

性中心. 首先, 反应物中的羟基氢原子转移到 GO 表面 与环氧官能团发生开环反应生成二醇, 然后发生脱水, 最后环氧官能团能够在氧气作用下恢复结构, 形成氧化 还原循环(如图 3 所示). 进一步调控含氧官能团的种类 和含量也可以改进 $\mathrm{GO}$ 的催化性能, Zhu 等 ${ }^{[68]}$ 通过连续 的酸和碱处理来合成改性 GO (abGO), 这种方法除去了 GO 表面不稳定的含氧基团, 增加了酚羟基的含量. abGO 可以作为苯甲醇氧化反应的催化剂, 苯甲醛的选 择性可达 $100 \%$, 产率随着 $\mathrm{abGO}$ 表面酚羟基的数量增 加呈现线性提高, 表明其表面酚羟基可能是反应的活性 位点. 作者根据 Eley-Rideal 机理解释: GO 表面的酚羟 基能够吸附和活化 $\mathrm{O}_{2}$ 形成活性氧, 活性氧可以直接进 攻苯甲醇中的羟基 $\mathrm{H}$ 原子形成苯甲醛并释放出 $\mathrm{H}_{2} \mathrm{O}$. 该 研究为 GO 表面官能团种类的控制提供了有效策略, 也 为此类催化剂的性能调控提供了重要技术参考.

通过在石墨烯中掺杂氮原子也可以提高其对苯甲 醇氧化反应的催化性能. Long 等 ${ }^{[69]}$ 发现高温法制备的 N 掺杂的石墨烯片(NG-T)可以催化苯甲醇的氧化反应. 通 过对不同温度下制备的样品进行分析, 发现催化剂中
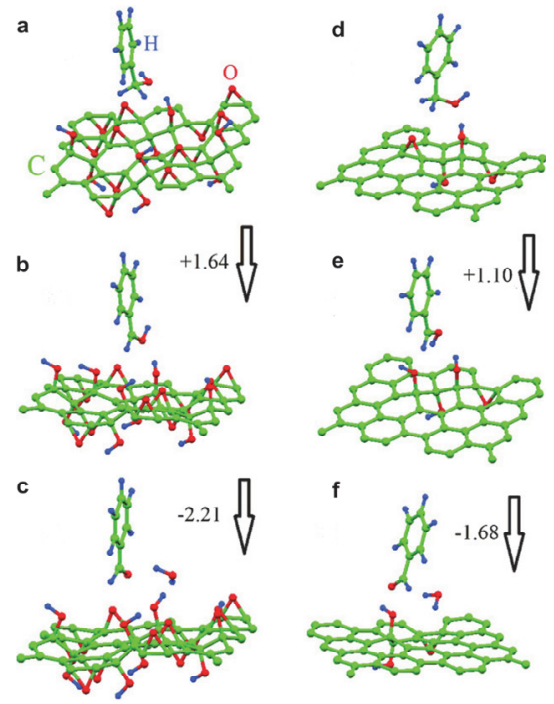

图 3 含有环氧基和羟基的 $\mathrm{GO}$ 催化苯甲醇氧化反应的过程描述, 反 应初始( $a, d)$, 中间 (b, e) 和最终 (c, f)步骤的优化原子结构图, GO 材料 平面上碳原子的含量为 $75 \%(\mathrm{a} \sim \mathrm{c})$ 或 $12.5 \%(\mathrm{~d} \sim \mathrm{f})^{[67]}$

Figure 3 Optimized atomic structures for the initial (a, d), intermediate $(b, e)$ and final (c, f) steps of benzyl alcohol oxidation over GO with the initial coverage of epoxy and hydroxyl groups, in which $75 \%(\mathrm{a} \sim \mathrm{c})$ or $12.5 \%(\mathrm{~d} \sim \mathrm{f})$ of the carbon atoms are on the material's basal plane ${ }^{[67]}$ 
$\mathrm{sp}^{2}$ 杂化的石墨 $\mathrm{N}$ 物种是氧化反应的催化活性中心, 石 墨 $\mathrm{N}\left(\mathrm{N}_{3} \mathrm{C}\right)$ 含量与初始反应速率之间存在良好的线性关 系(如图 4a)， $\mathrm{O}_{2}$ 在石墨 $\mathrm{N}$ 上吸附和活化有利于 $\mathrm{sp}^{2} \mathrm{~N}-$ $\mathrm{O}_{2}$ 加合物过渡态的形成, 反应可能机理如图 4b. 同样 地, 这一 $\mathrm{N}$ 掺杂活化策略也可以适用于 $\mathrm{B}$ 掺杂, $\mathrm{Yu}$ 等 ${ }^{[70]}$ 制备了 B 掺杂石墨烯(BG)并实现苯甲醇的选择性氧化 制备苯甲醛. 在石墨烯碳骨架中引入第二种杂原子还可 能进一步提高石墨烯材料的催化活性, 如 S、O 共掺杂 的多孔石墨烯显示了比 NG-T 更好的催化活性. Fan 等[71]通过热解硫酸盐木质素制备了 $\mathrm{S} 、 \mathrm{O}$ 共掺杂的多孔 石墨烯(G1000), 将其用于苯甲醇的催化氧化反应中, 取得优异的转化率和产率. 此外, G1000 对各种芳香醇 和 5-甲基糠醇等化合物也具有良好催化氧化活性, 显示 良好的底物普适性. DFT 计算表明, $\mathrm{C}-\mathrm{S}-\mathrm{C}$ 键作为苯 甲醇吸附和催化氧化的活性位点, 苯甲醇可以通过与 $\mathrm{S} 、 \mathrm{O}$ 共掺杂的石墨烯形成 $\mathrm{S}-\mathrm{O}$ 键, 较强的化学作用有 利于苯甲醇的吸附和活化. 吸附在 G1000 表面的苯甲 醇, 首先通过 $\mathrm{C}-\mathrm{H}$ 键脱氢生成 $\mathrm{C}_{6} \mathrm{H}_{5} \mathrm{CHOH}^{*}$, 然后通过 $\mathrm{O}-\mathrm{H}$ 键连续脱氢形成 $\mathrm{C}_{6} \mathrm{H}_{5} \mathrm{CHO}^{*}$ ，同时脱除的 $\mathrm{H}^{*}$ 原子 与 $\mathrm{O}^{*}$ (来自 $\mathrm{O}_{2}$ 裂解)结合以产生水. 苯甲醇在 $\mathrm{S} 、 \mathrm{O}$ 共掺 杂石墨烯上的两步连续脱氢的活化能分别为 $1.47 \mathrm{eV}$ 和 $0.86 \mathrm{eV}$ (如图 4c 所示), 远低于其在石墨烯实现这一过程 的活化能: $2.81 \mathrm{eV}$ 和 $1.72 \mathrm{eV}$ (如图 $4 \mathrm{~d}$ 所示), 使 $\mathrm{S} 、 \mathrm{O}$ 共 掺杂石墨烯具有较强的催化性能. 这些研究从分子和原 子尺度剖析石墨烯衍生物与反应底物的相互作用, 对于 无金属催化行为和机理的探究是至关重要的, 也可为高 性能石墨烯基碳基催化剂的设计和制备提供新的思路.

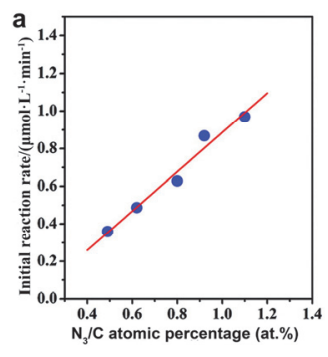

b

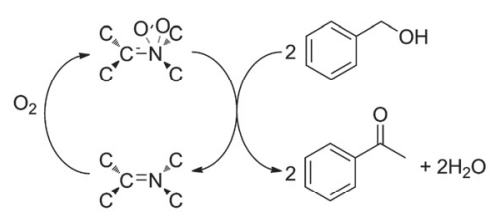

C
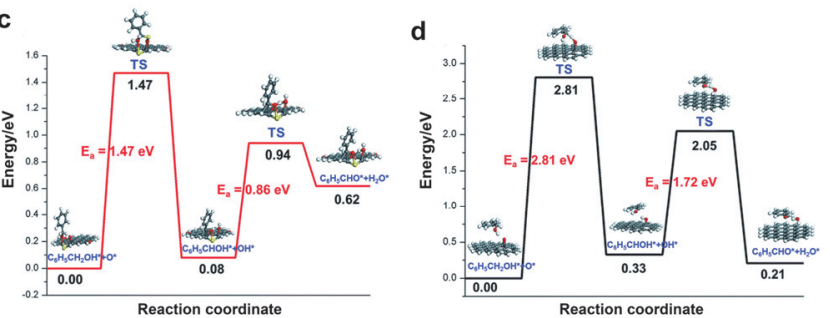

图 4 (a)初始反应速率与石墨氮含量的关系; (b) NG-T 催化苄醇的氧 化反应路径 ${ }^{69]}$; 利用 DFT 计算的石墨烯(c)和 S、O 共掺杂石墨烯(d) 模型催化苯甲醇氧化制苯甲醛的势能分布 ${ }^{[71]}$

Figure 4 (a) Relationship between initial reaction rate and graphitic $\mathrm{N}$ species; (b) proposed reaction pathway for benzyl alcohol oxidation catalyzed by NG-T ${ }^{[69]}$; potential energy profiles of benzyl alcohol oxidation to benzaldehyde over $\mathrm{S}$ and $\mathrm{O}$ co-doped graphene (c) and graphene (d) models by DFT calculations ${ }^{[71]}$
除了催化醇类化合物的选择性氧化之外，石墨烯类 催化剂的应用可拓展至惰性分子的氧化反应中. Ma 等 ${ }^{[55]}$ 报道了氧化还原法制备的石墨烯(CCG)可以催化双 氧水氧化苯为苯酚. $\mathrm{CCG}$ 的 $\pi$ 共轭结构在反应中充当 $\mathrm{H}_{2} \mathrm{O}_{2}$ 的活化位点, 且苯与 $\mathrm{CCG}$ 之间的 $\pi-\pi$ 相互作用使 得苯可以吸附在催化剂上, 促进反应的进行. 通过对比 $\mathrm{Pt} / \mathrm{SiO}_{2} 、 \mathrm{Mn} / \mathrm{CCG} 、 \mathrm{GO}$ 等催化剂的性能，作者发现 CCG 对 $\mathrm{H}_{2} \mathrm{O}_{2}$ 适宜的活化率、良好的苯吸附能力以及对氧化 反应的平衡动力学控制, 使其可以成功实现苯一步氧化 为苯酚. 由于芳环的稳定性较强, 决定了氧化反应发生 的难度较大, 因此, 该催化反应的转化率还有待进一步 提高. 相较于芳烃, 非芳香烃基的氧化反应则相对较为 容易发生. Xiao 等 ${ }^{[72]}$ 报道了 GO 催化环已烷的氧化制备 环己酮和环己醇, $\mathrm{GO}$ 的催化活性可与金属催化剂謧美. 作者对 GO 进行羧基化处理，随着处理时间增加 GO 片 中羧基化程度提高, 活性相应显著提高(如图 5a 所示), 表明 GO 表面的羧基可以促进反应的进行. 反应机理如 图 5b 所示, 羧基是氧化反应的活性位点, $\mathrm{O}_{2}$ 通过氢键与 羧基相互作用转化为 $\cdot \mathrm{O}^{2-}$, 然后 $\cdot \mathrm{O}^{2-}$ 可以引起自由基链 式反应, 夺取环已烷中 $\mathrm{H}$ 生成环己基过氧化氢(CHHP), 而 CHHP 在 GO 表面 $\mathrm{sp}^{2}$ 杂化体系中被分解, 得到环己 酮、环己醇和苯甲酸. 石墨烯基碳材料还可以将甲苯等 甲基取代芳烃氧化为相应的羧酸, Heidari 及其同事 ${ }^{[73]}$ 报 道了纳米氧化石墨烯片 $(\mathrm{NGO})$ 催化氧化二甲苯和甲苯 为相应的羧酸，二甲苯的转化率可以达到 $70 \%$, 产物对 苯二甲酸的选择性可以达到 $99 \%$.

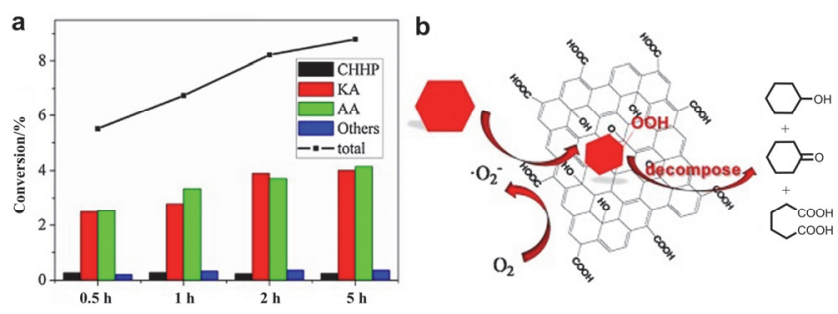

图 5 (a) 羧基含量对环己烷氧化的影响, (b) GO 催化氧化环己烷的可 能机理 ${ }^{[72]}$

Figure 5 (a) The effect of carboxyl acid group content on cyclohexane oxidation; (b) a possible mechanism of the cyclohexane oxidation with GO as the catalyst ${ }^{[72]}$

石墨烯基碳材料对茮基中 $\mathrm{C}-\mathrm{H}$ 键的氧化也有良好 的催化性能. $\mathrm{Ma}$ 及其同事 ${ }^{[74]}$ 报道了 $\mathrm{N}$ 掺杂的石墨烯 (LC-N)可以高效激活茮基的 $\mathrm{C}-\mathrm{H}$ 键, 催化氧化乙苯为 苯乙酮. 催化剂的催化活性随着 $\mathrm{N}$ 含量的增加而逐渐提 高, 当 $\mathrm{N}$ 含量为 $8.9 \%(w)$ 时, 乙苯的转化率可以达到 $98.6 \%$, 苯乙酮的产率为 $91.3 \%$. 此外, 作者发现催化剂 的活性也随着催化剂中石墨 $\mathrm{N}$ 的含量增加而增强. 通过 $\mathrm{C}$ 的 $\mathrm{K}$ 边 $\mathrm{X}$ 射线吸收光谱表征和 DFT 计算发现, $\mathrm{N}$ 掺杂 可以促进过氧化物类物种的形成, 而过氧化物类物种能 够激活 $\mathrm{C}-\mathrm{H}$ 键. 反应中石墨 $\mathrm{N}$ 与氧化剂并不直接形成 化学键, 而是通过掺杂到石墨烯中的石墨 $\mathrm{N}$ 改变其周围 
碳原子的电子云密度, 使其成为吸附过氧化氢等活性氧 物种的位点, 掺杂氮原子和其周围的碳原子共同构成了 催化反应活性中心. $\mathrm{Ma}$ 等 ${ }^{[75]}$ 还进一步探究了氮掺杂石 墨烯(NG)对烯烃的高效催化环氧化反应. 随着 NG 中石 墨 $\mathrm{N}$ 的比例增加(如图 6a), 催化活性也随之增强. 作者 的研究结果表明, 掺杂的石墨氮改变了周围碳原子的电 子环境, 使相邻的碳更容易形成活性氧物种. DFT 计算 结果显示 $\mathrm{NG}$ 中 $\mathrm{N}$ 的邻位碳是类过氧化物物种 $\cdot \mathrm{OOH}$ 最 稳定的吸附位置, 而且反应底物二苯乙烯或苯乙烯可以 通过 $\pi-\pi$ 相互作用吸附在石墨烯来促进反应发生, 反应 机理如图 $6 \mathrm{~b}$ 所示. $\cdot \mathrm{OOH}$ 中的 $\alpha$-氧攻击碳碳双键使得 - $\mathrm{O}-\mathrm{OH}$ 键被拉长, 而含有 $\beta$-氧的末端差基向另一个相 邻的碳迁移, 形成了环氧化物. 此外, 通过氧化剂叔丁 基过氧化氢和反应后催化剂表面羟基之间的反应, 活性 氧物种 $\cdot \mathrm{OOH}$ 得到恢复, 实现反应循环. 由此可见, 杂 原子的掺杂量和掺杂类型均对掺杂石墨烯的催化性能 有重要的影响.
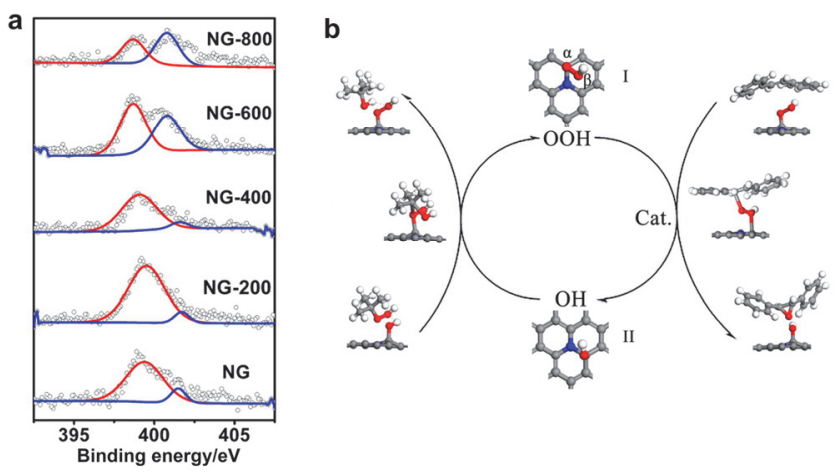

图 6 (a) 不同处理温度下 NG 的 N 1s 的 XPS 光谱; (b) NG 催化反式 二苯乙烯环氧化反应机理 ${ }^{[75]}$

Figure 6 (a) XPS spectra of N 1s of NG at different temperatures; (b) the reaction mechanism for the epoxidation of trans-stilbene on NG catalyst ${ }^{[75]}$

\section{2 还原反应和氢化反应}

石墨烯基催化剂在还原反应中应用也较为常见, 如 硝基芳烃的还原和炔烃半氢化反应等 ${ }^{[100]}$. Gao 等 ${ }^{[76]}$ 发 现 $\mathrm{rGO}$ 在室温下可以催化水合肼对硝基苯的还原反应, 苯胺的产率高达 $97.4 \%$, 其性能与贵金属催化剂 $\mathrm{Pt} / \mathrm{SiO}_{2}$ 相当. rGO 的锯齿形边缘具有独特的电子结构功能, 可 以作为催化反应活性中心, 促进反应的进行. DFT 计算 表明, rGO 边缘的碳原子通过与硝基苯中的氧原子相互 作用来削弱 $\mathrm{N}-\mathrm{O}$ 键, 从而活化硝基苯分子, 促进反应 进行. $\mathrm{N}$ 掺杂也有利于提高石墨烯材料对硝基芳烃催化 的还原活性, Yang 等 ${ }^{[77]}$ 通过 GO 和尿素水热法和热处理 制备了 $\mathrm{NG}$, 实现硝基芳烃的室温下高效催化还原. 反 应历程应是还原剂和硝基芳烃吸附在 NG 表面, 首先芳 香族硝基化合物被还原为亚硝基化合物中间体，再还原 为相应的羟胺. 然后, 一个亚硝基化合物分子和一个羟 胺分子反应生成氧化偶氮化合物, 再通过一系列步骤还
原为偶氮、肼和苯胺化合物. 反应物在 $\mathrm{NG}$ 催化剂表面 的吸附是催化反应的关键步骤，根据 DFT 计算表明，底 物在石墨 $\mathrm{N}$ 的吸附能最低，形成的 $\mathrm{N}-\mathrm{O}$ 键最长，石墨 烯中掺杂 $\mathrm{N}$ 能够激活周围的碳原子, 促进反应的进行. 这是由 $\mathrm{C}$ 和 $\mathrm{N}$ 元素的电负性差异造成的.

为了进一步提高 $\mathrm{NG}$ 的催化性能, 本课题组从增加 杂原子含量和构筑石墨烯片纵向传质孔道的角度来提 高石墨烯衍生物的催化性能. 以过氧化氢为刻蚀剂、氨 水为掺杂剂, 采用一锅同步水热法制备了 $\mathrm{N}$ 掺杂多孔石 墨烯(NHG)碳催化剂[78]. 研究结果表明, NHG 在对硝基 苯酚催化氢化反应中表现出优良的有机催化活性, 转化 频率可以达到 $3.32 \times 10^{-2} \mathrm{~min}^{-1}$ ，远高于 $\mathrm{Pd} / \mathrm{C}$ 催化剂 (5\% (w)) 和其它先前报道的碳催化剂和许多贵金属基催 化剂. 将 NHG 的催化应用拓展至一系列不饱和含氮有 机化合物(芳硝基化合物和含氮染料分子)的催化氢化反 应中，展现出良好的底物普适性(如图 7 所示). 利用 DFT 计算研究了 NHG 中碳原子电荷分布的电子结构变 化及其催化增强作用，阐明了催化活性位点及其无金属 催化机理. 此外, 本课题组还尝试在石墨烯骨架中引入 氮和磷两种杂原子，并探索了双掺杂石墨烯对硝基芳烃 的催化性能 ${ }^{[79]}$. 以六氯环三磷腈为氮、磷源, 在惰性气
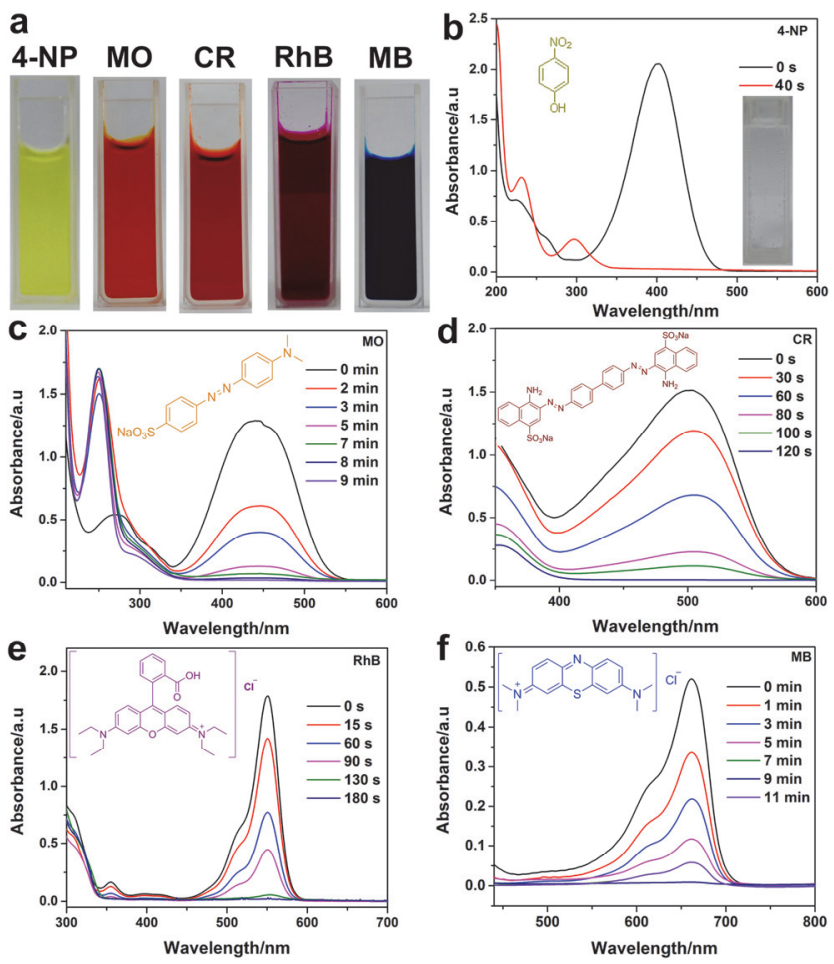

图 7 (a) 4-NP, MO, CR, RhB 和 MB 五种常见染料的水溶液实物照片; $(b \sim f)$ 无金属催化剂 NHG 催化 4-NP, MO, CR, RhB 和 $\mathrm{MB}$ 被 $\mathrm{NaBH}_{4}$ 还原的时间-紫外吸收强度图, 插图为相应染料分子的结构式; 图 7b 中插图为对 4-NP 还原后的溶液实物照片 ${ }^{[78]}$

Figure 7 (a) Photograph of the five common organic dyes (4-NP, MO, $\mathrm{CR}, \mathrm{RhB}$ and $\mathrm{MB}$ ) in aqueous solution; time-dependent absorption spectra showing the hydrogenation of 4-NP (b), MO (c), CR (d), RhB (e) and MB (f) using NHG as metal-free catalyst in the presence of $\mathrm{NaBH}_{4}$, insets are structural formula of the corresponding dye molecules. The inset of Fig. $7 \mathrm{~b}$ is the photograph of the reduced 4-NP solution ${ }^{[78]}$ 
体保护条件下与 rGO 共混退火进行 N/P 共掺杂，制备了 新型氮磷共掺杂多层石墨烯(NPG)(如图 8 所示). 研究 结果表明, 该催化剂在硝基芳烃催化还原反应中表现出 优良的催化活性、循环稳定性和底物普适性. 通过比较 催化剂 4-硝基苯酚(4-NP)的吸附和催化作用的速度, 发 现还原的速度比吸附的速度快, 即该反应为接触式反应 (图 8a, 8b). 利用 DFT 进行计算(图 $8 \mathrm{c} \sim 8 \mathrm{f}$ ), 探索了 N/P 共掺杂对石墨烯中碳原子电荷分布的调控规律及其催 化增强作用(或协同增强作用), 阐明了 NPG 的无金属催 化机理, 进而为石墨烯基催化剂的性能提高提供了理论 参考.
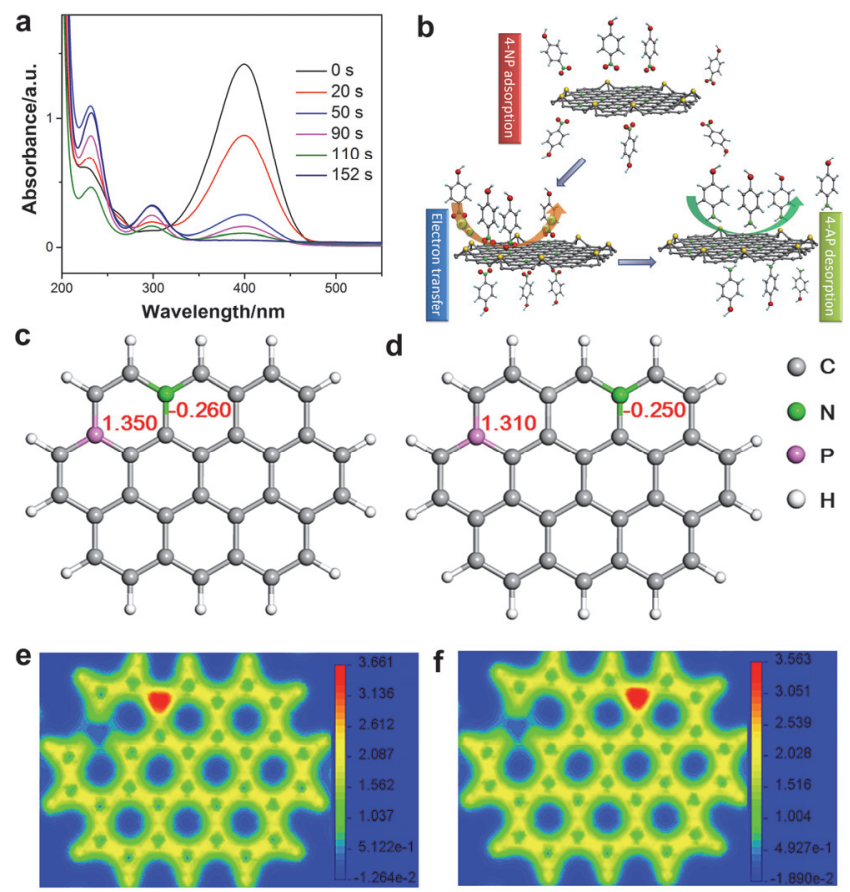

图 8 (a) 氮磷共掺杂石墨烯催化对硝基苯酚还原为对氨基苯酚时间紫外吸收强度图; (b) 氮磷共掺杂石墨烯的催化机理图; (c, d) NPG 的 两种结构模型的电荷密度, (e, f) 两种模型相应的电子密度分布图 [79]

Figure 8 (a) Time-dependent UV-vis spectra recording the catalytic conversion of 4-NP to 4-AP using NPG as the carbocatalyst; (b) proposed reaction pathway of 4-NP reduction on NPG. Analog structure of (c, d) two NPG models and their charge densities. (e, f) Total electron density distribution mapping of the two NPG models ${ }^{[79]}$

除了可以用于硝基芳烃的还原反应外, 无金属石墨 烯基催化材料也可以促进乙烯和乙炔等不饱和烃的氢 化反应. Primo 等[80]发现单层石墨烯可以作为烯烃选择 性加氢的催化剂, 石墨烯可以高效和高选择性地催化乙 烯气流中的乙炔 $\left(n\left(\mathrm{C}_{2} \mathrm{H}_{4}\right): n\left(\mathrm{C}_{2} \mathrm{H}_{2}\right)=9: 1\right)$ 的半氢化反 应，乙炔的转化率高达 99\%. 石墨烯材料表面的 Lewis 酸碱对作为反应的活性中心, 可以激活吸附在表面的 $\mathrm{H}_{2}$ 形成 $\mathrm{H}^{+}$和 $\mathrm{H}^{-}$活性氢, 随后转移到含有碳碳三键的底 物上. 然而, 此半氢化反应停留在乙烯阶段的机理应与 贵金属的部分毒化减活(如 Lindar 催化剂)完全不同, 具 体催化机制还有待深入研究和阐明.

\section{3 偶联反应}

偶联反应是有机合成中用于构建碳碳键的高效策 略之一，此类反应通常采用贵金属钯作为其催化剂. 近 年来, 研究者发现石墨烯基催化剂对碳碳偶联反应也有 良好的催化活性，利用其替代金属钯不仅可以降低合成 成本，还可以避免金属残留的问题 ${ }^{[101]}$. $\mathrm{Ma}$ 等 ${ }^{[81]}$ 报道了 以 $\mathrm{GO}$ 催化苯环中 $\mathrm{C}-\mathrm{H}$ 键的活化, 使得芳基碘代物与 苯等芳烃发生直接烷基化反应生成联芳基化合物. 研究 结果显示叔丁醇钾对反应的进行至关重要, GO 中的含 氧基团可通过稳定和激活 $\mathrm{K}^{+}$离子来促进反应进行. $\mathrm{K}^{+}$ 离子能容易地激活碘苯的 $\mathrm{C}-\mathrm{I}$ 键形成 $\mathrm{C}_{6} \mathrm{H}_{5}$ • 自由基中间 体, $\mathrm{C}_{6} \mathrm{H}_{5} \bullet$ 和苯通过与 $\mathrm{GO}$ 的 $\pi-\pi$ 相互作用吸附在 $\mathrm{GO}$ 表 面，进而发生偶联生成联芳基化合物(如图 9). 联芳基化 合物也可以通过 GO 催化的直接脱氢偶联反应来制备, Gong 等 ${ }^{[82]}$ 报道了 GO 催化的芳烃直接脱氢偶联反应. 以 1,3,5-三甲氧基苯为底物, 乙腈为溶剂, 在 $\mathrm{GO}$ 催化下 联芳基化合物的产率可达到 86\%. 此外, 研究表明 GO 对大多数的 $\beta$-䒬酚衍生物、 $\beta$-䒬胺衍生物均具有良好的 催化偶联活性, 说明其对芳烃直接脱氢偶联反应具有良 好的底物适用性. 作者认为 GO 片层中未成对电子促进 了偶联反应的进行，反应中底物以自由基方式实现连 接.

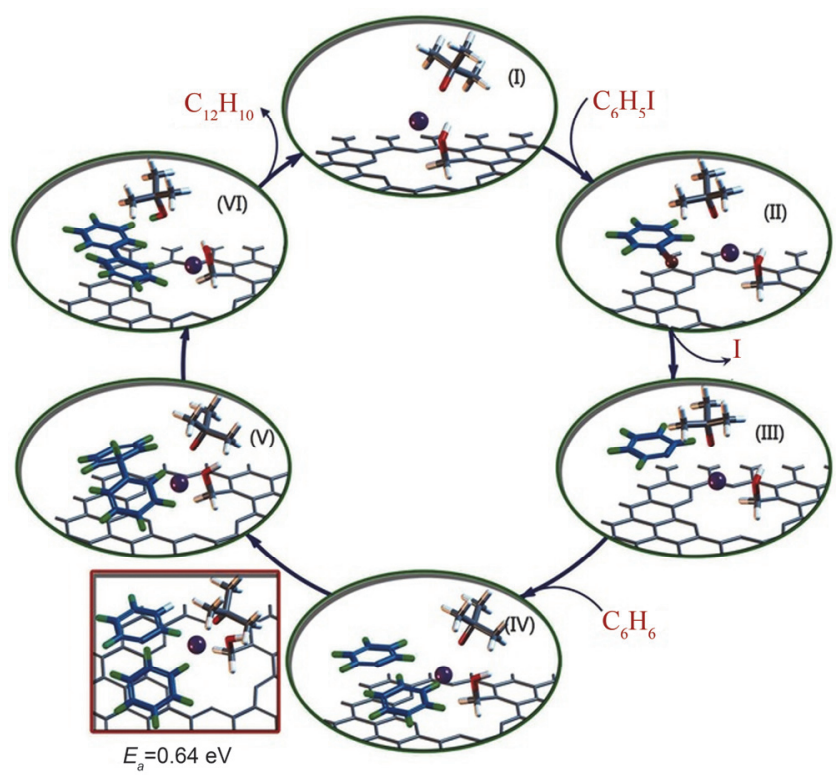

图 9 GO 催化芳基化反应的机理 ${ }^{[81]}$

Figure 9 Mechanism for the GO-catalyzed arylation reaction ${ }^{[81]}$

除了催化形成碳-碳键的偶联反应之外，石墨烯基 材料也可催化碳-氮键的构建, 如催化胺类化合物的氧 化自偶联反应制备亚胺 ${ }^{[102-103]}$. Huang 等 ${ }^{[83]}$ 报道了氧化 石墨可以催化茮胺及其衍生物的 $\mathrm{O}_{2}$ 氧化偶联反应，将 其转化为相应的亚胺, 产率可达 98\%. 在 2012 年, $\mathrm{Su}$ 等 ${ }^{84]}$ 先后用碱和酸对 $\mathrm{GO}$ 进行处理来增加 GO 中的缺陷 位, 制备了一种新的催化剂 ba-GO, 并在茮胺的催化氧 
化偶联反应中展现出优异的催化活性. 通过酸碱处理可 以激活 $\mathrm{GO}$ 中的催化位点, 使得 ba-GO 中缺陷边缘的羧 基以及局域未配对电子在反应中可以协同作用捕获分 子氧和胺分子. 催化机理如图 10 所示, 茮胺先通过氢键 与缺陷边缘羧基结合, ba-GO 中的未成对电子还原 $\mathrm{O}_{2}$ 形 成 $\cdot \mathrm{O}_{2}^{-}, \cdot \mathrm{O}_{2}^{-}$再从胺基化反应中获取氢原子生成亚胺中 间体, 最后亚胺中间体与游离的胺偶联生成产物. 催化 剂在 6 次循环反应后, 亚胺的产率并没有明显变化, 显 示了良好的稳定性. Yang 等[85]发现对石墨烯进行 P 掺杂 改性也可以提升其催化性能, 制备的磷掺杂石墨烯(PG) 可以实现催化茮胺的无金属氧化偶联反应生成 $N$-芐叉 苯胺, 产率可达 $82 \%$, 远高于 $\mathrm{NG}$ 和石墨烯的催化产率 (53\%和 45\%). 作者利用 DFT 探究了反应历程, 计算结 果表明茮胺分子在 $\mathrm{PG}$ 基体吸附能最低, $\mathrm{O}_{2}$ 吸附在 $\mathrm{PG}$ 上 时 $\mathrm{O}-\mathrm{O}$ 键的键长被拉长, 增强了 $\mathrm{O}_{2}$ 分子的活性, 有利 于形成超氧自由基 $\cdot \mathrm{O}^{2-}$, 随后苄胺的氧化反应通过 $\cdot \mathrm{O}^{2-}$ 自由基路径进行. 此外, 通过在石墨烯骨架中引入两种 杂原子也可以提高其催化氧化性能, 如 Antonietti 等 ${ }^{[62]}$ 利用 $\mathrm{B}$ 和 $\mathrm{N}$ 共掺杂调控石墨烯的导带和价带, 实现胺的 氧化偶联制备亚胺类化合物.

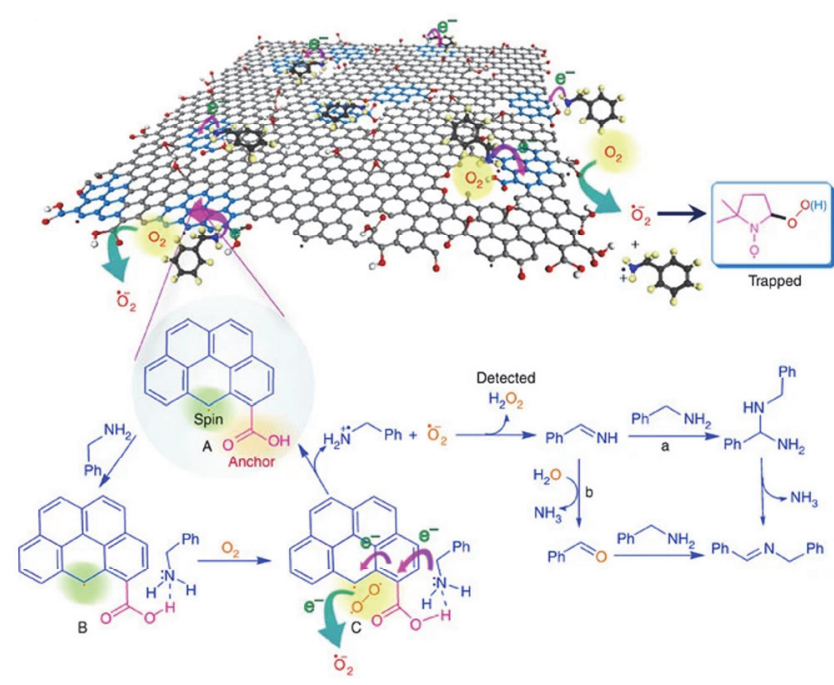

图 10 ba-GO 催化伯胺氧化偶联的机理 ${ }^{[8]}$

Figure 10 Mechanism of ba-GO catalysed oxidative coupling of primary amines ${ }^{[84]}$

\section{4 取代反应}

取代反应可分为亲电取代反应和亲核取代反应，如 Friedel-Crafts 反应和羧酸衍生物的酯化/酯交换反应等. Friedel-Crafts 反应是制备芳香族化合物的重要方式, 可 以分为烷基化反应和酰基化反应. Szostak 等 ${ }^{[86]}$ 报道了 $\mathrm{GO}$ 催化芳烃与苯乙烯/醇的 Friedel-Crafts 烷基化, 可以 高效和高选择性地在芳环上引入相应的烷基，1,3-二甲 氧基苯与苯乙烯的烷基化反应产率高达 $98 \%$. 研究结果 显示, 以醇为底物时, 醇与催化剂表面的含氧官能团发 生瞬时配位而被激活. 以烯烃为底物时, 反应通过串联
机制进行，烯烃先发生水合反应转化为醇，再发生取代 反应，其中水合是该反应的关键步骤. GO 表面的含氧 基团作为反应的活性中心，通过与两个反应底物的相互 作用直接促进芳环上 Friedel-Crafts 烷基化反应(如图 11). 除了催化制备联芳基烷烃化合物外, GO 对非苯芳 烃的 Friedel-Crafts 烷基化反应也有良好的催化效果. Wang 及其同事 ${ }^{[87]}$ 报道了 GO 可以催化醛和吲哚的 Friedel-Crafts 烷基化反应，制备双吲哚甲烷. GO 表面的 大量羧基可作为反应的活性中心加强羟基碳亲电性，从 而实现醛与吲哚的 Friedel-Crafts 烷基化反应，生成双吲 哚甲烷. Peng 等 ${ }^{[88}$ 报道了类似的反应，他们以 GO 作为 催化剂实现了吲哚与醚的 Friedal-Craft 反应, 合成了 3,3'-双吲哚甲烷衍生物. 与 Friedel-Crafts 烷基化相比, 石墨烯基催化剂在 Friedel-Crafts 酰基化反应中的应用 相对较少. 1997 年, Kodomari 等 ${ }^{[89]}$ 报道了石墨能促进苯 甲醚、甲苯和邻二甲苯等芳香族化合物和酰卤化物发生 Friedel-Crafts 酰化反应，制备芳基酮.
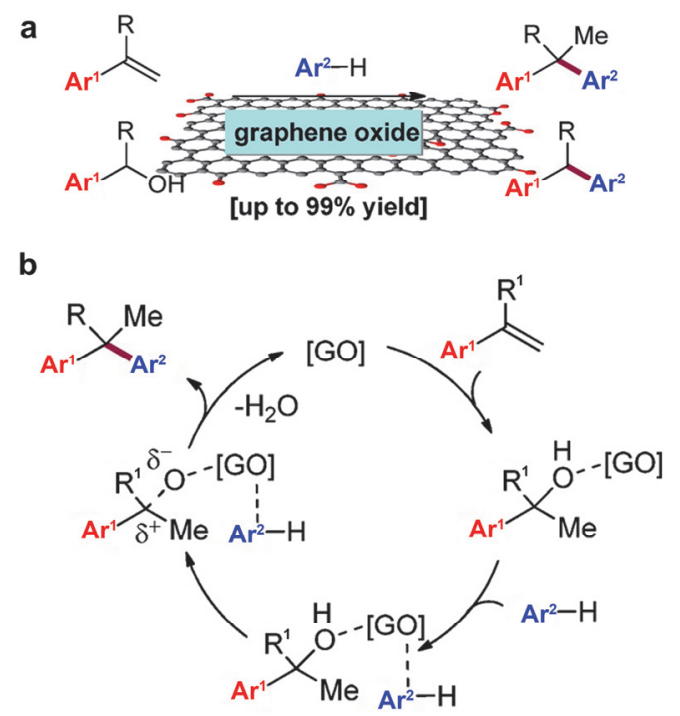

图 11 (a) GO 催化 Friedel-Crafts 烷基化反应; (b) GO 催化芳烃 Friedel-Crafts 烷基化反应的机理 ${ }^{[86]}$

Figure 11 (a) GO-Catalyzed Friedel-Crafts alkylation reaction; (b) mechanism for the GO-catalyzed alkylation of arenes ${ }^{[86]}$

此外, 在石墨烯基材料上引入强酸性官能团亦可进 一步提高其酸性，并拓展其催化应用范围，如，适用于 脂肪酸的酯化反应和植物油的酯交换反应. Nongbe 等[90]使用对氨基苯磺酸对 $\mathrm{rGO}$ 进行功能化修饰制备了 磺化石墨烯 $\left(\mathrm{GR}-\mathrm{SO}_{3} \mathrm{H}\right)$ 固体酸催化剂, 并成功催化棕㭣 油与甲醇进行酯交换反应制备生物柴油，石墨烯片表面 连接的苯磺酸基团作为催化活性中心. GR- $\mathrm{SO}_{3} \mathrm{H}$ 在棕㭣 油的酯交换反应中展现了良好的催化活性和稳定性，反 应 $14 \mathrm{~h}$ 后，生物柴油的产率达到 $78 \%$ 。催化剂经过 4 次 连续循环使用后，生物柴油的产率仅有微弱降低，表明 该催化剂具有较强的耐用性. Zhang 等[91]报道了采用改 进的 Hummers 法制备了一种富硫氧化石墨烯(GO-S)磺 
酸/羧酸双元酸催化剂, 成功催化油酸与甲醇的酯化反 应制备生物柴油. GO-S 表面的- $\mathrm{COOH}$ 基团和 $-\mathrm{SO}_{3} \mathrm{H}$ 之 间的协同作用增强了 “复合酸体系” 在酯化反应中的 催化活性. 这些由石墨烯材料催化的无金属反应为油脂 类精细化学品的合成提供了绿色合成方案.

\section{5 其他反应}

除上述常见的几种催化反应类型之外, 石墨烯基无 金属催化剂的应用领域也不断在拓展, 研究人员发现其 在其他有机反应中具有良好的催化性能，如 aldol 反应、 Aza-Michael 加成反应以及 Knoevenagel 缩合反应等. Jia 等 ${ }^{[92]}$ 报道了氧化石墨可以作为苯乙炔和苯乙醇自动串 联氧化-水合-羟醛偶联反应的催化剂, 以苯乙炔和苯乙 醇为底物一锅法制备查尔酮. 氧化石墨先催化苯乙炔发 生水合反应生成苯乙酮, 苯甲醇氧化为苯甲醛, 再催化 苯乙酮和苯甲醛的 aldol 反应得到查尔酮. Ma 及其同 事 ${ }^{[93]}$ 也报道了二乙烯三胺修饰的 GO (GO-DETA) 可以 作为查尔酮和丙二腈 Michael 加成反应、苯甲醛和丙二 腈 Knoevenagel 缩合反应的催化剂. 经过二乙烯三胺修 饰的 GO 表面具有伯、仲氨基和羧基三种基团，三者协 同作用促进了反应的发生. Knoevenagel 缩合反应中, GO-DETA 表面的羧基和伯氨基与苯甲醛反应形成亚胺 阳离子中间体, 然后仲氨基与丙二腈反应生成丙二腈阴 离子, 再进攻亚胺阳离子中间体形成最终产物. 此外, 查尔酮和丙二腈也可通过类似机理发生 Michael 加成反 应。

GO 表面或边缘含有大量的羧基等含氧基团, 使其 呈现一定的酸性, 赋予其良好的有机催化性能. Zhao 等 ${ }^{[94]}$ 报道了以 $\mathrm{GO}$ 为催化剂催化纤维素水解为葡萄糖, 纤维素转化率 $30.2 \%$, 葡萄糖产率可达 $21.4 \%$. GO 的准 均匀柔性层状结构有利于邻接的羧基和酚羟基在水解 反应中的协同作用, 使 GO 展现出比氧化石墨、传统固 体酸更好的催化性能. Gong 课题组利用 GO 表面的大量 含氧官能团催化了胺的 $N$-甲酰化反应 ${ }^{[95]}$, 含氮杂环化 合物的脱氢反应 ${ }^{[96]}$ 以及芳烃及酮的氧化碘化、双碘化反

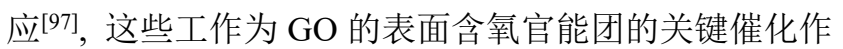
用提供了重要的理论参考. 得益于这些含氧官能团, 石 墨类碳材料还可以作为分子间脱水反应的催化剂. Bielawski 等 ${ }^{[98]}$ 报道了氧化石墨表面的含氧官能团可以 催化苯甲醇的脱水, 进而促进聚合反应的发生制备苯撑 亚甲基复合材料. Xiao 等[99]报道石墨烯可催化室温下乙 醇和硝酸盐中 $\mathrm{C}-\mathrm{C}$ 和 $\mathrm{N}-\mathrm{O}$ 键断裂形成 $\mathrm{C} \equiv \mathrm{N}$ 键, 该 无金属催化反应为腈的制备提供了新的路径. 总之, 石 墨烯衍生物参与的有机反应随着无金属碳基催化的研 究还在进一步拓展和深入, 将会作为金属催化领域的重 要补充.

\section{4 总结与展望}

本综述首先介绍了石墨烯基碳材料出色的物理和
化学特性, 然后总结了石墨烯基碳材料作为无金属催化 剂，在氧化、还原、氢化、偶联、取代和其他有机反应 中的应用，并相应总结了可能的活性位点和反应机理， 展现了石墨烯基催化材料在有机化学反应中的良好应 用潜力. 随着绿色化学和可持续发展等创新理念的持续 和深化, 石墨烯基碳材料作为催化剂在过去的十几年中 引起了科研人员的广泛研究兴趣, 然而, 无金属碳基催 化的研究仍然处于初级阶段, 还需要更多的深入研究来 推进该领域的持续发展.

石墨烯基无金属催化剂及其在有机催化应用研究 领域的发展还需要注意以下几点: (1)首先，无金属碳基 催化剂的应用范围还具有一定的局限性，难以像金属基 催化剂那样普遍适用于大多数有机反应中; (2)其次, 目 前的研究对催化反应的活性位点和机理的研究仍不够 透彻, 不利于石墨烯基催化剂的设计和制备, 这从某种 程度上限制了石墨烯基催化剂的发展，因此这有赖于表 征技术不断进步和研究工作的持续深入开展; (3)再次， 大部分碳基催化剂的性能还不够强，使得一些有机催化 反应中的催化剂投料量较大, 这需要研究者探究石墨烯 材料修饰或者掺杂的新技术，以便进一步提高催化剂的 催化活性; (4)最后，石墨烯基催化剂在有机催化中的应 用主要在实验室条件下开展, 虽然研究现状展现了良好 的应用前景，却鲜有工业应用的报道，这就要求石墨烯 基催化剂完成工业催化案例，积累更多的实际应用经 验.

\section{作者简介}

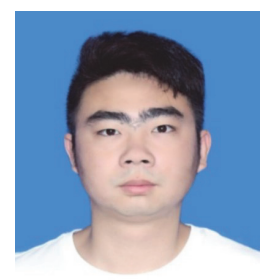

黄杰, 2019 年本科毕业于武汉工程大学, 目前在武汉工程 大学化学与环境工程学院柏正武课题组开展研究工作, 研究 方向是碳基催化剂在有机反应中的应用.

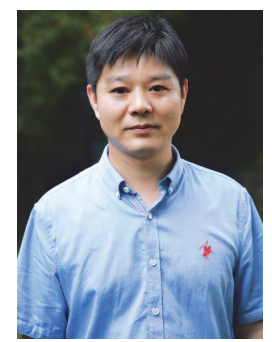

奚江波, 博士、副教授、硕士生导师. 主要研究领域涉及 高性能贵金属纳米催化剂、单原子催化剂和无金属碳基催化 剂的制备及其在有机催化、电催化、生物传感等方面的应用, 以及催化机理的研究. 近年来在 Advanced Functional Materials、Applied Catalysis B: Environmental、Journal of Catalysis 
等国际期刊上发表了学术论文 30 余篇, 获授权中国发明专利 5 项.

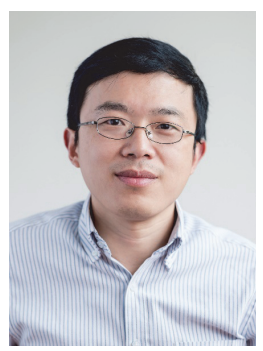

陈伟, 博士, 副教授, 硕士生导师. 主要研究领域涉及无 机材料的合成、表征与应用, 并从事手性分离材料的研究及色 谱分析工作. 主持湖北省教育厅科学技术研究计划重点项目 1 项, 在国内外学术期刊上发表科研论文 20 余篇, 其中 SCI 收 录论文 7 篇, 授权发明专利 1 项.

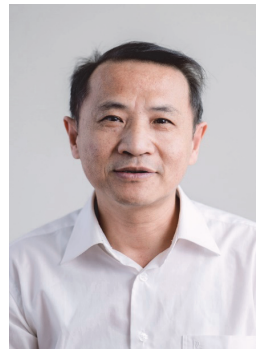

柏正武, 博士、教授、博士生导师. 研究领域涉及有机合 成、药物研制及手性功能材料的制备与应用. 主持并完成了 4 项国家自然科学基金资助的面上项目, 取得了一些有自主知 识产权的研究成果, 获得多项发明专利权, 在国际学术期刊 上发表研究论文 60 余篇.

\section{References}

[1] Chowdhury, A. D.; Houben, K.; Whiting, G. T.; Chung, S.-H.; Baldus, M.; Weckhuysen, B. M. Nat. Catal. 2018, 1, 23.

[2] Bakandritsos, A.; Kadam, R. G.; Kumar, P.; Zoppellaro, G.; Medved, M.; Tucek, J.; Montini, T.; Tomanec, O.; Andryskova, P.; Drahos, B.; Varma, R. S.; Otyepka, M.; Gawande, M. B.; Fornasiero, P.; Zboril, R. Adv. Mater. 2019, 31, 1900323.

[3] Zou, Y. J.; Cheng, H. J.; Wang, H. N.; Huang, R. X.; Xu, Y. H.; Jiang, J.; He, Q.; Liu, C. H.; Liu, J. C.; Xiong, J. M.; Yao, J. N.; Huangfu, X. L.; Ma, J. Environ. Sci. Technol. 2020, 54, 7205.

[4] Pan, L.; Xu, M.-Y.; Feng, L.-J.; Chen, Q.; He, Y.-J.; Han, B.-H. Polym. Chem. 2016, 7, 2308.

[5] Martin-Aranda, R. M.; Cejka, J. Top. Catal. 2010, 53, 141.

[6] Nakagawa, K. J. Jpn. Pet. Inst. 2019, 62, 53.

[7] Pentsak, E. O.; Cherepanova, V. A.; Ananikov, V. P. ACS Appl. Mater. Interfaces 2017, 9, 36723.

[8] Du, Z. T.; Shao, Z. H. Chem. Soc. Rev. 2013, 42, 1337.

[9] Hu, H. W.; Xin, J. H.; Hu, H.; Wang, X. W.; Kong, Y. Y. Appl. Catal. A-Gen. 2015, 492, 1.

[10] Pandey, R. K.; Prajapati, V. K. Int. J. Biol. Macromol. 2018, 107, 1278.

[11] Polshettiwar, V.; Varma, R. S. Green Chem. 2010, 12, 743.

[12] Su, D. S.; Wen, G. D.; Wu, S. C.; Peng, F.; Schlogl, R. Angew. Chem. Int. Ed. 2017, 56, 936

[13] Su, D. S.; Zhang, J.; Frank, B.; Thomas, A.; Wang, X. C.; Paraknowitsch, J.; Schlogl, R. ChemSusChem 2010, 3, 169.

[14] Su, D. S.; Perathoner, S.; Centi, G. Chem. Rev. 2013, 113, 5782.

[15] Centi, G.; Perathoner, S.; Su, D. S. Catal. Surv. Asia 2014, 18, 149.

[16] Geim, A. K.; Novoselov, K. S. Nat. Mater. 2007, 6, 183.

[17] Novoselov, K. S.; Geim, A. K.; Morozov, S. V.; Jiang, D.; Zhang, Y.; Dubonos, S. V.; Grigorieva, I. V.; Firsov, A. A. Science 2004, 306,
666.

[18] Chua, C. K.; Pumera, M. Chem. - Eur. J. 2015, 21, 12550.

[19] Balandin, A. A.; Ghosh, S.; Bao, W. Z.; Calizo, I.; Teweldebrhan, D.; Miao, F.; Lau, C. N. Nano Lett. 2008, 8, 902.

[20] Stoller, M. D.; Park, S. J.; Zhu, Y. W.; An, J. H.; Ruoff, R. S. Nano Lett. 2008, 8, 3498.

[21] Chua, C. K.; Pumera, M. Chem. Soc. Rev. 2014, 43, 291.

[22] Huang, C. S.; Li, Y. J.; Wang, N.; Xue, Y. R.; Zuo, Z. C.; Liu, H. B.; Li, Y. L. Chem. Rev. 2018, $118,7744$.

[23] Zuo, Z. C.; Li, Y. L. Joule 2019, 3, 899

[24] Du, Y. C.; Zhou, W. D.; Gao, J.; Pan, X. Y.; Li, Y. L. Acc. Chem. Res. 2020, $53,459$.

[25] Hui, L.; Xue, Y. R.; Yu, H. D.; Liu, Y. X.; Fang, Y.; Xing, C. Y.; Huang, B. L.; Li, Y. L. J. Am. Chem. Soc. 2019, 141, 10677.

[26] Xue, Y. R.; Huang, B. L.; Yi, Y. P.; Guo, Y.; Zuo, Z. C.; Li, Y. J.; Jia, Z. Y.; Liu, H. B.; Li, Y. L. Nat. Commun. 2018, 9, 1460.

[27] Li, L.; Zuo, Z. C.; Wang, F.; Gao, J. C.; Cao, A. M.; He, F.; Li, Y. L. Adv. Mater. 2020, 32, 2000140.

[28] Roy, A. S.; Poulose, A. C.; Bakandritsos, A.; Varma, R. S.; Otyepka, M. Appl. Mater. Today 2021, 23, 101053.

[29] Li, X. T.; Wang, J.; Duan, X. G.; Li, Y.; Fan, X. B.; Zhang, G. L.; Zhang, F. B.; Peng, W. C. ACS Catal. 2021, 11, 4848.

[30] Park, M.; Lee, J.; Kim, B. S. Nanoscale 2021, 13, 10143.

[31] Ahmad, M. S.; Nishina, Y. Nanoscale 2020, 12, 12210.

[32] Wang, Z. Y.; Pu, Y.; Wang, D.; Wang, J. X.; Chen, J. F. Front. Chem. Sci. Eng. 2018, 12, 855.

[33] Liu, J. Q.; Tang, J. G.; Gooding, J. J. J. Mater. Chem. 2012, 22, 12435.

[34] Daelemans, B.; Bilbao, N.; Dehaen, W.; De Feyter, S. Chem. Soc. Rev. 2021, 50, 2280

[35] Srivastava, S. K.; Pionteck, J. J. Nanosci. Nanotechnol. 2015, 15, 1984.

[36] Dreyer, D. R.; Todd, A. D.; Bielawski, C. W. Chem. Soc. Rev. 2014, 43, 5288.

[37] Wang, X. W.; Sun, G. Z.; Routh, P.; Kim, D. H.; Huang, W.; Chen, P. Chem. Soc. Rev. 2014, 43, 7067.

[38] Dimiev, A. M.; Tour, J. M. ACS Nano 2014, 8, 3060.

[39] Rosillo-Lopez, M.; Lee, T. J.; Bella, M.; Hart, M.; Salzmann, C. G. RSC Adv. 2015, 5, 104198.

[40] Wang, C. I.; Periasamy, A. P.; Chang, H. T. Anal. Chem. 2013, 85, 3263.

[41] Sun, L. Chin. J. Chem. Eng. 2019, 27, 2251.

[42] Dreyer, D. R.; Park, S.; Bielawski, C. W.; Ruoff, R. S. Chem. Soc. Rev. 2010, 39, 228.

[43] Lerf, A.; He, H.; Forster, M. Phys. Chem. B 1998, 102, 4477.

[44] Yang, J. H.;Yang, D.; Tang, P.; Ma, D. Acta Phys.-Chim. Sin. 2016, 32,75 (in Chinese). (杨敬贺, 杨朵, 唐沛, 马丁, 物理化学学报, 2016, 32, 75.)

[45] Loh, K. P.; Bao, Q. L.; Eda, G.; Chhowalla, M. Nat. Chem. 2010, 2, 1015.

[46] Wan, W. B.; Li, L. L.; Zhao, Z. B.; Hu, H.; Hao, X. J.; Winkler, D. A.; Xi, L. C.; Hughes, T. C.; Qiu, J. S. Adv. Funct. Mater. 2014, 24 4915.

[47] Eigler, S.; Hu, Y. C.; Ishii, Y.; Hirsch, A. Nanoscale 2013, 5, 12136.

[48] Wang, Y.; Li, S. S.; Yang, H. Y.; Luo, J. RSC Adv. 2020, 10, 15328.

[49] Su, C. L.; Loh, K. P. Acc. Chem. Res. 2013, 46, 2275.

[50] Feng, J. L.; Ye, Y. Q.; Xiao, M.; Wu, G.; Ke, Y. Chem. Pap. 2020, $74,3767$.

[51] Larciprete, R.; Fabris, S.; Sun, T.; Lacovig, P.; Baraldi, A.; Lizzit, S. J. Am. Chem. Soc. 2011, 133, 17315.

[52] Xu, C.; Yuan, R. S.; Wang, X. New Carbon Mater. 2014, $29,61$.

[53] Moon, I. K.; Lee, J.; Ruoff, R. S.; Lee, H. Nat. Commun. 2010, $1,1$.

[54] Navalon, S.; Dhakshinamoorthy, A.; Alvaro, M.; Garcia, H. Chem. Rev. 2014, 114, 6179

[55] Yang, J. H.; Sun, G.; Gao, Y. J.; Zhao, H. B.; Tang, P.; Tan, J.; Lu, A. H.; Ma, D. Energy Environ. Sci. 2013, 6, 793.

[56] Wang, Z.; Pu, Y.; Wang, D.; Wang, J.-X.; Chen, J.-F. Front. Chem. Sci. Eng. 2018, 12, 855 .

[57] Azlouk, M.; Durmaz, M.; Zor, E.; Bingol, H. Mater. Chem. Phys. 2020, 239, 122298

[58] Liu, H. T.; Liu, Y. Q.; Zhu, D. B. J. Mater. Chem. 2011, 21, 3335.

[59] Zhang, D. Y.; Lei, L. Y.; Shang, Y. H. Chem. Ind. Eng. Prog. 2016, 35, 831 (in Chinese). (张德懿，雷龙艳，尚永花，化工进展, 2016, 35,831 .)

[60] Zheng, Y.; Jiao, Y.; Jaroniec, M.; Jin, Y. G.; Qiao, S. Z. Small 2012, 
8,3550 .

[61] Feng, L. Y.; Qin, Z. Y.; Huang, Y. J.; Peng, K. S.; Wang, F.; Yan, Y. Y.; Chen, Y. G. Sci. Total Environ. 2020, 698, 134239.

[62] Li, X. H.; Antonietti, M. Angew. Chem. Int. Ed. 2013, 52, 4572.

[63] Patel, M. A.; Luo, F. X.; Khoshi, M. R.; Rabie, E.; Zhang, Q.; Flach, C. R.; Mendelsohn, R.; Garfunkel, E.; Szostak, M.; He, H. X. ACS Nano 2016, 10, 2305.

[64] Kong, X.-K.; Chen, C.-L.; Chen, Q.-W. Chem. Soc. Rev. 2014, 43, 2841.

[65] Guo, X. L.; Qi, W.; Liu, W.; Yan, P. Q.; Li, F.; Liang, C. H.; Su, D. S. ACS Catal. 2017, 7, 1424.

[66] Dreyer, D. R.; Jia, H.-P.; Bielawski, C. W. Angew. Chem., Int. Ed. 2010, 49, 6813.

[67] Boukhvalov, D. W.; Dreyer, D. R.; Bielawski, C. W.; Son, Y. W. ChemCatChem 2012, 4, 1844.

[68] Zhu, S. H.; Cen, Y. L.; Yang, M. A.; Guo, J.; Chen, C. M.; Wang, J. G.; Fan, W. B. Appl. Catal., B-Environ. 2017, 211, 89.

[69] Long, J. L.; Xie, X. Q.; Xu, J.; Gu, Q.; Chen, L. M.; Wang, X. X. ACS Catal. 2012, 2, 622.

[70] Cheng, W. J.; Liu, X. T.; Li, N.; Han, J. T.; Li, S. M.; Yu, S. S. RSC $A d v$. 2018, 8, 11222.

[71] Zhu, S. H.; Chen, Y. Y.; Gao, X. Q.; Lv, Z. X.; He, Y.; Wang, J. G.; Fan, W. B. Catal. Sci. Technol. 2020, 10, 2786.

[72] Xiao, Y. P.; Liu, J. C.; Xie, K. H.; Wang, W. B.; Fang, Y. X. Mol. Catal. 2017, 431, 1.

[73] Heidari, M.; Sedrpoushan, A.; Mohannazadeh, F. Org. Process Res. Dev. 2017, 21, 641

[74] Gao, Y. J.; Hu, G.; Zhong, J.; Shi, Z. J.; Zhu, Y. S.; Su, D. S.; Wang, J. G.; Bao, X. H.; Ma, D. Angew. Chem. Int. Ed. 2013, 52, 2109.

[75] Li, W. J.; Gao, Y. J.; Chen, W. L.; Tang, P.; Li, W. Z.; Shi, Z. J.; Su, D. S.; Wang, J. G.; Ma, D. ACS Catal. 2014, 4, 1261.

[76] Gao, Y. J.; Ma, D.; Wang, C. L.; Guan, J.; Bao, X. H. Chem. Commun. 2011, 47, 2432.

[77] Yang, F.; Chi, C.; Wang, C. X.; Wang, Y.; Li, Y. F. Green Chem. 2016, 18, 4254.

[78] He, Z. L.; Liu, J.; Wang, Q. J.; Zhao, W.; Wen, Z. P.; Chen, J.; Manoj, D.; Xie, C. Y.; Xi, J. B.; Yu, J. X.; Tang, C. Y.; Bai, Z. W.; Wang, S. J. Catal. 2019, 377, 199.

[79] Xi, J. B.; Wang, Q. J.; Liu, J.; Huan, L.; He, Z. L.; Qiu, Y.; Zhang, J.; Tang, C. Y.; Xiao, J.; Wang, S. J. Catal. 2018, 359, 233.

[80] Primo, A.; Neatu, F.; Florea, M.; Parvulescu, V.; Garcia, H. Nat. Commun. 2014, 5, 5291

[81] Gao, Y. J.; Tang, P.; Zhou, H.; Zhang, W.; Yang, H. J.; Yan, N.; Hu, G.; Mei, D. H.; Wang, J. G.; Ma, D. Angew. Chem., Int. Ed. 2016, 55, 3124.
[82] Fang, J. X.; Peng, Z. Y.; Yang, Y.; Wang, J. W.; Guo, J. Y.; Gong, H. Asian J. Org. Chem. 2018, 7, 355.

[83] Huang, H.; Huang, J.; Liu, Y. M.; He, H. Y.; Cao, Y.; Fan, K. N. Green Chem. 2012, 14,930.

[84] Su, C. L.; Acik, M.; Takai, K.; Lu, J.; Hao, S. J.; Zheng, Y.; Wu, P. P.; Bao, Q. L.; Enoki, T.; Chabal, Y. J.; Loh, K. P. Nat. Commun. 2012, 3, 1 .

[85] Yang, F.; Fan, X. X.; Wang, C. X.; Yang, W.; Hou, L. Q.; Xu, X. W.; Feng, A. D.; Dong, S.; Chen, K.; Wang, Y.; Li, Y. F. Carbon 2017, $121,443$.

[86] Hu, F.; Patel, M.; Luo, F. X.; Flach, C.; Mendelsohn, R.; Garfunkel, E.; He, H. X.; Szostak, M. J. Am. Chem. Soc. 2015, 137, 14473.

[87] Wang, Y. H.; Sang, R.; Zheng, Y.; Guo, L.; Guan, M.; Wu, Y. Catal. Commun. 2017, 89, 138.

[88] Peng, X. J.; Zen, Y.; Liu, Q.; Liu, L. X.; Wang, H. S. Org. Chem. Front. 2019, 6, 3615.

[89] Kodomari, M.; Suzuki, Y.; Yoshida, K. Chem. Commun. 1997, 1567.

[90] Nongbe, M. C.; Ekou, T.; Ekou, L.; Yao, K. B.; Le Grognec, E.; Felpin, F. X. Renew. Energ. 2017, 106, 135.

[91] Zhang, H. L.; Luo, X.; Shi, K. Q.; Wu, T.; He, F.; Zhou, S. B.; Chen, G. Z.; Peng, C. ChemSusChem 2017, 10, 3352

[92] Jia, H. P.; Dreyer, D. R.; Bielawski, C. W. Adv. Synth. Catal. 2011, 353, 528.

[93] Yang, A. W.; Li, J. J.; Zhang, C.; Zhang, W. Q.; Ma, N. Appl. Surf. Sci. 2015, 346, 443 .

[94] Zhao, X. C.; Wang, J.; Chen, C. M.; Huang, Y. Q.; Wang, A. Q.; Zhang, T. Chem. Commun. 2014, 50, 3439.

[95] Ma, J.; Zhang, J. Y.; Zhou, X.; Wang, J. W.; Gong, H. J. Iran. Chem. Soc. 2018, 15, 2851.

[96] Zhang, J. Y.; Chen, S. Y.; Chen, F. F.; Xu, W. S.; Deng, G. J.; Gong, H. Adv. Synth. Catal. 2017, 359, 2358.

[97] Zhang, J. Y.; Li, S. G.; Deng, G. J.; Gong, H. ChemCatChem 2018, $10,376$.

[98] Dreyer, D. R.; Jarvis, K. A.; Ferreira, P. J.; Bielawski, C. W. Macromolecules 2011, 44, 7659.

[99] Xiao, D.; Wang, W. C.; Gai, Y. Z.; Zhao, Y. Sci. Rep. 2018, 8, 1750.

[100] Tang, P.; Hu, G.; Li, M. Z.; Ma, D. ACS Catal. 2016, 6, 6948.

[101] Wen, G. D.; Gu, Q. Q.; Liu, Y. F.; Schlogl, R.; Wang, C. X.; Tian, Z. J.; Su, D. S. Angew. Chem. Int. Ed. 2018, 57, 16898.

[102] Chen, B.; Wang, L. Y.; Gao, S. ACS Catal. 2015, 5, 5851.

[103] Mukherjee, A.; Nerush, A.; Leitus, G.; Shimon, L. J. W.; Ben David, Y.; Jalapa, N. A. E.; Milstein, D. J. Am. Chem. Soc. 2016, 138, 4298.

(Cheng, B.) 